\title{
Poincaré series of curves on rational surface singularities
}

\author{
A. Campillo*, F. Delgado*and S. M. Gusein-Zade**
}

\begin{abstract}
For a reducible curve singularity embedded in a rational surface singularity the Poincaré series is computed. Here the Poincaré series is defined by the multi-index filtration on the local ring defined by orders of a function on the branches of the curve. The method of the computations is based on the notion of the integral with respect to the Euler characteristic over the projectivization of the ring of functions (notion similar to, and inspired by, the notion of motivic integration). For the case of the $\mathbf{E}_{8}$ surface singularity it appears that the Poincare series coincides with the Alexander polynomial of the corresponding link.
\end{abstract}

Mathematics Subject Classification (2000). 14H20, 14J17, 32S25.

Keywords. Poincaré series, curve singularities, rational surfaces, Alexander polynomial.

In [3] and [4] there was computed the Poincaré series (in several variables) of the multiindex filtration on the ring of germs of functions of two variables defined by orders of a function on the branches of a reducible plane curve singularity $(C, 0) \subset\left(\mathbb{C}^{2}, 0\right)$. It was shown that this Poincaré series coincides with the Alexander polynomial (in several variables) of the link of the singularity $(C, 0)$. In [5] there was computed the Poincare series of the multi-index filtration on the ring of germs of functions on a rational surface singularity $(\$, 0)$ defined by the multiplicities of a function along components of the exceptional divisor of a resolution of the singularity $(\$, 0)$. The method of the computations is based on the notion of the integral with respect to the Euler characteristic over the projectivization of the ring of functions. This notion is similar to (and inspired by) the notion of motivic integration.

The Poincare series of a plane curve singularity is computed in terms of an embedded resolution of the curve. The answer is tightly connected with the Poincaré series of the set of divisorial valuations corresponding to the resolution. A generalization of this approach for a twisted (i.e., non plane) curve would be to consider the curve being embedded into a surface singularity and to use its embedded resolution. The Poincaré series of the set of divisorial valuations of a resolution of a surface singularity is well understood only for rational ones. Therefore it is natural to consider curves

\footnotetext{
*The first two authors were partially supported by the grant MCyT BFM2001-2251.

** Partially supported by the grants RFBR-04-01-00762, INTAS-00-259.
} 
on them. Here we apply the mentioned method of computing the Poincare series to a (reducible) curve on a rational surface singularity.

It appears that curves on the $\mathbf{E}_{8}$ surface singularity have special properties. The link of the surface singularity $\mathbf{E}_{8}$ is a homology sphere. Therefore, for a curve on the $\mathbf{E}_{8}$ surface singularity, there is defined the Alexander polynomial (in several variables) of the corresponding link. We show that it also coincides with the Poincare series of the curve singularity.

\section{Poincaré series of a curve on a rational surface singularity}

Let $(C, 0)$ be a (in general, reducible) germ of a curve and let $C=\bigcup_{j=1}^{r} C_{j}$ be its decomposition into irreducible components (with a fixed numbering). Let $\varphi_{j}:(\mathbb{C}, 0) \rightarrow(C, 0)$ be an uniformization of the branch $C_{j}, j=1, \ldots, r$. For a germ $g \in \mathcal{O}_{C, 0}$, let $w_{j}=w_{j}(g)$ be the power of the leading term in the power series decomposition of the germ $g \circ \varphi_{j}:(\mathbb{C}, 0) \rightarrow \mathbb{C}: g \circ \varphi_{j}(\tau)=a \cdot \tau^{w_{j}}+$ terms of higher degree $(a \neq 0)$. If $g \circ \varphi_{j}(\tau) \equiv 0$ we assume $w_{j}(g)$ to be equal to $+\infty$. Let $\underline{w}(g):=\left(w_{1}(g), \ldots, w_{r}(g)\right)$ (we call $\underline{w}(g)$ the value of the function $g$ on the curve $C)$. For $\underline{w}=\left(w_{1}, \ldots, w_{r}\right) \in \mathbb{Z}^{r}$, let $J(\underline{w})=\left\{g \in \mathcal{O}_{C, 0}: w_{j}(g) \geq w_{j}\right.$, $j=1, \ldots, r\}\left(J(\underline{w})\right.$ is an ideal in $\left.\mathcal{O}_{C, 0}\right)$, and let $c(\underline{w}):=\operatorname{dim} J(\underline{w}) / J(\underline{w}+\underline{1})$, where $\underline{1}=(1, \ldots, 1), L_{C}\left(t_{1}, \ldots, t_{r}\right)=\sum_{\underline{w} \in \mathbb{Z}^{r}} c(\underline{w}) \cdot \underline{\underline{w}}$ (here $\underline{t} \underline{\underline{w}}=t_{1}^{w_{1}} \ldots t_{r}^{w_{r}}$, pay attention that the sum is over all $\underline{w}$ in $\mathbb{Z}^{r}$, not only over positive ones).

The Poincare series of the multi-index filtration defined by $\underline{w}(\bullet)$ (for short the Poincare series of the curve $(C, 0)$ ) is the power series (in fact a polynomial for $r \geq 2$ ):

$$
P_{C}\left(t_{1}, \ldots, t_{r}\right)=\frac{L_{C}\left(t_{1}, \ldots, t_{r}\right) \cdot \prod_{j=1}^{r}\left(t_{j}-1\right)}{t_{1} \ldots t_{r}-1} .
$$

Remark. If the curve $(C, 0)$ is embedded into an ambient space $(X, 0)$, in the definition of the Poincaré series $P_{C}\left(t_{1}, \ldots, t_{r}\right)$, one can use the ring $\mathcal{O}_{X, 0}$ of germs of functions on $(X, 0)$ instead of $\mathcal{O}_{C, 0}$ above.

From now on let the curve singularity $(C, 0)$ be embedded into a rational surface singularity $(8,0)$. In [5] there was defined the notion of the integral with respect to the Euler characteristic over the projectivization $\mathbb{P} \mathcal{O}_{s, 0}$ of the ring of germs of the functions on the surface $(\$, 0)$ (see also [2], [4]). Just as in [4], [5] one can show that

$$
P_{C}\left(t_{1}, \ldots, t_{r}\right)=\int_{\mathbb{P} \mathcal{O}_{\$, 0}} \underline{t}^{\underline{w}(g)} d \chi,
$$

where $\underline{t} \underline{w}(g)$ is a function on $\mathbb{P} \mathcal{O}_{s, 0}$ with the values in the abelian group (with respect to the addition) $\mathbb{Z}\left[\left[t_{1}, \ldots, t_{r}\right]\right], t_{i}^{\infty}$ is assumed to be equal to 0 . 
Let $\pi:(\mathcal{X}, \mathscr{D}) \rightarrow(\$, 0)$ be an embedded resolution of the curve $C \subset \&$, i.e., a resolution of the surface singularity $(\&, 0)$ such that $\pi^{-1}(C)$ is a normal crossing divisor. Let $\widetilde{C}_{j}$ be the strict transform of the component $C_{j}$ of the curve $C$ ( $j=$ $1, \ldots, r)$. Let $E_{i}, i=1, \ldots, s$, be the irreducible components of the exceptional divisor $\mathscr{D}=\pi^{-1}(0)$. All the components $E_{i}$ are isomorphic to the projective line $\mathbb{C P}^{1}$. Let $-k_{i}$ be the self intersection number $E_{i} \circ E_{i}$ of the component $E_{i}$. Let $M=-\left(E_{i} \circ E_{\ell}\right)$ be minus the intersection matrix of the components of the exceptional divisor $\mathscr{D}$. The matrix $M$ has numbers $k_{i}$ on the diagonal, other entries of $M$ are equal to -1 or 0 .

For a function $g \in \mathcal{O}_{s, 0}, g \neq 0$, let $v_{i}(g)(i=1, \ldots, s)$ be the multiplicity of the lifting $g \circ \pi$ of the function $g$ along the component $E_{i}\left(v_{i}(\bullet)\right.$ is the divisorial valuation corresponding to the component $\left.E_{i}\right)$. Let $\underline{v}(g)=\left(v_{1}(g), \ldots, v_{s}(g)\right)$. The set $S_{\S}$ of points of the lattice $\mathbb{Z}_{\geq 0}^{s}$ of the form $\underline{v}(g)$ is a subsemigroup of $\mathbb{Z}_{\geq 0}^{s}$ (the semigroup of divisorial valuations of the resolution $\pi$ ). The semigroup $S_{\text {\& }}$ consists of all $\underline{v} \in \mathbb{Z}_{\geq 0}^{S}$ such that $\underline{v} M \geq 0$ (i.e., such that

$$
k_{i} v_{i}-\sum_{\ell: E_{i} \cap E_{\ell}=p t} v_{\ell} \geq 0
$$

for $i=1, \ldots, s$; see e.g [8]). Let $m=\operatorname{det}(M), A=M^{-1}=\left(a_{i j}\right)$, and let $\underline{a}_{i}=\left(a_{i 1}, \ldots, a_{i s}\right)$ (note that the determinant $m$ does not depend on the resolution $\bar{\pi}$ of $(\&, 0)$ and that $m a_{i j}$ are integers). The set $\underline{v} M \geq 0$ in $\mathbb{R}^{s}$ is the simplicial cone generated by the vectors $\underline{a}_{i}, i=1, \ldots, s$. One can show (see [8]) that $\underline{a}_{i}>0$, $i=1, \ldots, s$.

Remark. Let $n_{i}=n_{i}(\underline{v})$ be the left hand sides of the inequalities (1), i.e., $\underline{n}=\left(n_{1}, \ldots, n_{s}\right)=\underline{v} M$ (in particular $\left.n_{i}\left(\underline{a}_{j}\right)=\delta_{i j}\right)$. One can easily see that, for $\underline{v}=\underline{v}(g) \in S_{8}, n_{i}(\underline{v})$ is equal to the intersection number of the strict transform of the curve $\{g=0\}$ with the component $E_{i}$ of the exceptional divisor. Let $\underline{v}(\underline{n}):=\underline{n} M^{-1}$.

Let ${\stackrel{\circ}{E_{i}}}_{\text {(respectively }} \dot{E}_{i}$ ) be the "smooth part" of the component $E_{i}$ in the total transform of the curve $C$ (respectively in the exceptional divisor $\mathscr{D}$ ), i.e., $E_{i}$ minus intersection points with all other components of the total transform $\pi^{-1}(C)$ of the curve $C$ (respectively of the exceptional divisor $\mathcal{D}$ ). The divisorial valuations $v_{i}$ define a multi-index filtration on the ring $\mathcal{O}_{\$, 0}$ of functions on the surface singularity $(8,0)$. Let $P_{\S, \pi}\left(T_{1}, \ldots, T_{s}\right)$ be the Poincare series of this filtration defined in the same way as above (it depends on the surface singularity $\&$ and on its resolution $\pi$ ).

For a fractional power series $Q\left(T_{1}, \ldots, T_{s}\right) \in \mathbb{Z}\left[\left[T_{1}^{1 / m}, \ldots, T_{s}^{1 / m}\right]\right]$, let Int $Q\left(T_{1}, \ldots, T_{s}\right)$ be its "integer part", i.e., the sum of all the monomials from $Q\left(T_{1}, \ldots, T_{s}\right)$ with integer exponents. The main result of [5] (formulated in somewhat different terms) is the following. 
Theorem 1. For a rational surface singularity $(\mathcal{S}, 0)$ and a resolution $\pi:(\mathcal{X}, \mathbb{D}) \rightarrow$ $(\$, 0)$, one has

$$
P_{\S, \pi}\left(T_{1}, \ldots, T_{s}\right)=\operatorname{Int} \prod_{i=1}^{s}\left(1-\underline{T}^{a_{i}}\right)^{-\chi\left(\dot{E}_{i}\right)} .
$$

Now we give a formula for the Poincaré series of the curve $(C, 0) \subset(\S, 0)$.

Theorem 2. One has

$$
P_{C}\left(t_{1}, \ldots, t_{r}\right)=\left.\left(\operatorname{Int} \prod_{i=1}^{s}\left(1-\underline{T}^{\underline{a}_{i}}\right)^{-\chi\left(\stackrel{\circ}{E_{i}}\right)}\right)\right|_{T_{i} \mapsto \prod_{j: \tilde{c}_{j} \cap E_{i}=p t} t_{j}}
$$

(in the substitution above $\prod_{j \in \emptyset} t_{j}$ is supposed to be equal to 1 ).

Proof. For a topological space $X$, let $S^{n} X=X^{n} / S_{n}$ be the $n$th symmetric power of the space $X$. Let

$$
Y=\bigcup_{\underline{n} \in \mathbb{Z}_{\geq 0}^{s}}\left(\prod_{i=1}^{s} S^{n_{i}} \stackrel{\circ}{E_{i}}\right)=\prod_{i=1}^{s}\left(\bigcup_{n=0}^{\infty} S^{n} \stackrel{\circ}{E}_{i}\right)
$$

and

$$
Y_{0}=\bigcup_{\underline{n} \in \mathbb{Z}_{\geq 0}^{s}: \underline{v}(\underline{n}) \in \mathbb{Z}^{s}}\left(\prod_{i=1}^{s} S^{n_{i}} \stackrel{\circ}{E_{i}}\right) .
$$

Elements of $Y$ and of $Y_{0}$ are represented by collections of points (finite sets of points with multiplicities) of the smooth part $\stackrel{\circ}{D}=\bigcup_{i=1}^{s} \stackrel{\circ}{E_{i}}$ of the exceptional divisor $\mathscr{D}$ (for elements of $Y_{0}$ with an additional condition). For a function $g \in \mathcal{O}_{s, 0}$ such that the strict transform of its zero level curve $\{g=0\}$ intersects the exceptional divisor $\mathscr{D}$ only at smooth points (i.e., at points of $\stackrel{\circ}{\mathscr{D}}$ ), the collection of intersection points counted with multiplicities (intersection numbers) belongs to $Y_{0}$. Moreover, a divisor on $\mathcal{X}$ which intersects the exceptional divisor $\mathscr{D}$ only at smooth points is the strict transform of the zero level curve of a function if and only if the collection of the intersection points of this divisor with $\mathcal{D}$ (counted with multiplicities) belongs to $Y_{0}$.

Let $\underline{v}$ be a function on $Y$ with values in $\mathbb{Q}_{\geq 0}^{s}$ which is equal to $\sum_{i=1}^{s} n_{i} \underline{a}_{i}$ on the component of $Y$ with the number $\underline{n}$. The values of the function $\underline{v}$ on the space $Y_{0} \subset Y$ belong to $\mathbb{Z}_{\geq 0}^{s}$ and, moreover, $\underline{v}^{-1}\left(\mathbb{Z}_{\geq 0}^{s}\right)=Y_{0}$. One has

$$
\int_{Y} \underline{T}^{\underline{v}} d \chi=\prod_{i=1}^{s}\left(1-\underline{T}^{\underline{a}_{i}}\right)^{-\chi\left(\stackrel{\circ}{E}_{i}\right)}
$$


(see, e.g., [4]; this follows from the formula $1+\chi(X) t+\chi\left(S^{2} X\right) t^{2}+\chi\left(S^{3} X\right) t^{3}+\cdots=$ $(1-t)^{-\chi(X)}$, where $\chi(X)$ is the Euler characteristic of the space $\left.X\right)$,

$$
\int_{Y_{0}} \underline{T}^{\underline{v}} d \chi=\operatorname{Int} \prod_{i=1}^{s}\left(1-\underline{T}^{\underline{a}_{i}}\right)^{-\chi\left(\stackrel{\circ}{E}_{i}\right)} .
$$

Let a map $\mathbb{Z}_{\geq 0}^{s} \rightarrow \mathbb{Z}_{\geq 0}^{r}\left(\mathbb{Q}_{\geq 0}^{s} \rightarrow \mathbb{Q}_{\geq 0}^{r}\right)$ be defined by $\left(v_{1}, \ldots, v_{s}\right) \mapsto\left(w_{1}, \ldots, w_{r}\right)$ with $w_{j}=v_{i(j)}$, where $i=i(j)$ is the number of the component $E_{i}$ of the exceptional divisor $\mathscr{D}$ which intersects the strict transform $\widetilde{C}_{j}$ of the component $C_{j}$ of the curve $C$. One has

$$
\int_{Y_{0}} \underline{t}^{\underline{w} \underline{(v)}} d \chi=\int_{Y_{0}} \underline{T}^{\underline{v}} d \chi \mid T_{i} \mapsto \prod_{j: \widetilde{C}_{j} \cap E_{i}=p t} t_{j}
$$

(and the same for the integrals over $Y$ ).

For a function $g \in \mathcal{O}_{s, 0}$ such that the strict transform of its zero level curve $\{g=0\}$ intersects the exceptional divisor $\mathscr{D}$ only at smooth points (and thus the collection of them belongs to the space $\left.Y_{0}\right)$ the value $\underline{w}(g)$ of the function $g$ on the curve $C$ is equal to $\underline{w}(\underline{v}(g))$.

Let $\underline{V} \in \mathbb{Z}_{>0}^{r}$. Making as many additional blow-ups of intersection points of components of the total transform of the curve $C$ as it is necessary, we can suppose that, for any $g \in \mathcal{O}_{\S, 0}$ with $\underline{w}(g) \leq \underline{V}$, the strict transform of the curve $\{g=0\}$ intersects the exceptional divisor $\mathcal{D}$ only at smooth points. Let $\mathbb{P} \mathcal{O}_{\S, 0}(\underline{V})$ be the set $\left\{g \in \mathbb{P} \mathcal{O}_{s, 0}: \underline{w}(g) \leq \underline{V}\right\}$. The $\operatorname{set} \mathbb{P} \mathcal{O}_{s, 0}(\underline{V})$ is cylindric (see, e.g., [5], Proposition 1). Let $I$ be the map from $\mathbb{P} \mathcal{O}_{s, 0}(\underline{V})$ to the space $Y_{0}$ which sends a class $g \in \mathbb{P} \mathcal{O}_{s, 0}(\underline{V})$ with $\underline{w}(g) \leq \underline{V}$ to the collection of intersection points of the strict transform of the curve $\{g=0\}$ with the exceptional divisor $\mathscr{D}$ (counted with multiplicities). One can easily see that $\underline{w}(I(g))=\underline{w}(g)$ (in fact also $\underline{v}(I(g))=\underline{v}(g)$ ). Moreover, the image $\operatorname{Im} I$ of the map $I$ coincides with the union $Y_{0}^{\underline{V}}$ of all the components of the space $Y_{0}$ with $\underline{w} \leq \underline{V}$. Preimages of points of the space $Y_{0}^{\underline{V}}$ under the map $I$ are complex affine spaces (see, e.g., [5] Proposition 2). Since the Euler characteristic of a complex affine space is equal to 1 , the Fubini formula (applied to the map $I: \mathbb{P} \mathcal{O}_{s, 0}(\underline{V}) \rightarrow Y_{0}^{\underline{V}}$ ) implies that

$$
\int_{Y_{0}^{\underline{V}}} \underline{t}^{\underline{w}} d \chi=\int_{\mathbb{P} \mathcal{O}_{s, 0}(\underline{V})} \underline{t}^{\underline{w}} d \chi .
$$

Since this equation holds for any $\underline{V} \in \mathbb{Z}_{\geq 0}^{r}$, one has

$$
\int_{Y_{0}} \underline{t} \underline{w} d \chi=\int_{\mathbb{P} \mathcal{O}_{8,0}} \underline{t}^{\underline{w}} d \chi=P_{C}(\underline{t}) .
$$




\section{Curves on the surface singularity $E_{8}$}

Let $(\&, 0)$ be the rational double point of the type $\mathbf{E}_{8}\left(\left\{x^{2}+y^{3}+z^{5}=0\right\} \subset\left(\mathbb{C}^{3}, 0\right)\right)$. In this case the determinant $m$ of the intersection matrix $M$ is equal to 1 . This implies that all the vectors $\underline{a}_{i}$ are integer and therefore any curve on $(\delta, 0)$ is the zero level curve of a function (i.e., each Weil divisor is a Cartier one). Therefore, for a curve $C=\bigcup_{i=1}^{r} C_{i} \subset(\&, 0)$, one has

$$
P_{C}\left(t_{1}, \ldots, t_{r}\right)=\left.\prod_{i=1}^{s}\left(1-\underline{T}^{\underline{a}_{i}}\right)^{-\chi\left(\dot{E}_{i}\right)}\right|_{T_{i} \mapsto \prod_{j: \widetilde{c_{j}} \cap E_{i}=p t} t_{j}} .
$$

The link $L=s \cap S_{\epsilon}^{5}$ (where $S_{\epsilon}^{5}$ is the sphere of small radius $\epsilon$ centered at the origin of $\mathbb{C}^{3}$ ) is a homology 3-sphere (see, e.g., [7]). For the curve $C$, let $K=S_{\epsilon}^{5} \cap C$ be the corresponding link. The manifold $K$ is the union of $r$ circles in the homology sphere $L$. Therefore there is defined the Alexander polynomial of $(L, K)$ which is a polynomial in $r$ variables (see, e.g., [6]). Let $\Delta^{\S, C}\left(t_{1}, \ldots, t_{r}\right)$ denote the Alexander polynomial of the pair $(L, K)$.

Let $\pi:(\mathcal{X}, \mathscr{D}) \rightarrow(\S, 0)$ be an embedded resolution of the curve $C \subset \&$. For a component $E_{i}$ of the exceptional divisor $\mathscr{D}=\pi^{-1}(0)$, let $\widetilde{L}$ be a germ of a smooth curve transversal to the component $E_{i}$ at a smooth point. Let the curve $L=\pi(\widetilde{L}) \subset(\$, 0)$ be defined by an equation $\{g=0\}$. From the remark on page 3 the following statement follows.

Lemma 1. One has $\underline{v}(g)=\underline{a}_{i}$.

From [6] it follows that

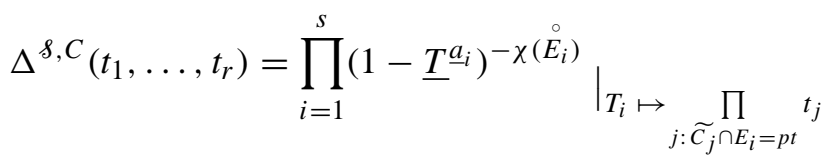

when $r>1$ and, for $r=1$,

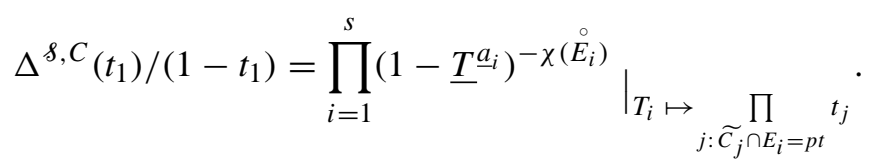

Note that the substitution in the last formula means that $T_{i} \mapsto 1$ if $\widetilde{C_{1}} \cap E_{i}=\emptyset$ and $T_{i} \mapsto t_{1}$ otherwise.

Remark. According to the general definition (see, e.g., [6]), the Alexander polynomial $\Delta^{S, C}\left(t_{1}, \ldots, t_{r}\right)$ of a link is well defined only up to multiplication by monomials $\pm \underline{t} \underline{\underline{m}}= \pm t_{1}^{m_{1}} \ldots t_{r}^{m_{r}}$ with $\underline{m}=\left(m_{1}, \ldots, m_{r}\right) \in \mathbb{Z}^{r}$. For the link $(L, K)=$ 
$(\&, C) \cap S_{\epsilon}^{5}$, the formulae (3) and (4) fixes the choice of the Alexander polynomial in such a way that it is really a polynomial (i.e., does not contain monomials with negative exponents) and its value at the origin $(\underline{t}=0)$ is equal to 1 .

Comparing (3) and (4) with (2) we have the following statement.

Theorem 3. Let $C=\bigcup_{i=1}^{r} C_{i}$ be a curve on the $\mathbf{E}_{8}$ surface singularity ( 8,0$)$. Then, if $r>1$, one has

$$
P_{C}\left(t_{1}, \ldots, t_{r}\right)=\Delta^{\&, C}\left(t_{1}, \ldots, t_{r}\right)
$$

and for the case $r=1$,

$$
P_{C}\left(t_{1}\right)=\Delta^{\&, C}\left(t_{1}\right) /\left(1-t_{1}\right) .
$$

Corollary. On the $\mathbf{E}_{8}$ surface singularity, there are only curves the Poincaré series of which are products/ratios of cyclotomic polynomials.

As an example, the curve given by $t \mapsto\left(t^{3}, t^{4}, t^{5}\right)$ does not lie on an $\mathbf{E}_{8}$ surface singularity.

Remark. Let $\&$ be an arbitrary rational surface singularity and let $\widetilde{P}_{\S, C}\left(T_{1}, \ldots, T_{s}\right)=$ $\prod_{i=1}^{s}\left(1-\underline{T}^{\underline{a}} \underline{ }^{-\chi}\left(\stackrel{\circ}{E}_{i}\right)\right.$ be the fractional power series corresponding to an embedded resolution $\pi:(\mathcal{X}, \mathcal{D}) \rightarrow(\&, 0)$ of the curve $C \subset(\&, 0)$. Suppose that the curve $C$ is a Cartier divisor, i.e., it is the zero level curve of a function $f \in \mathcal{O}_{\S, 0}$. Let $\zeta_{f}(t)$ be the zeta-function of the classical monodromy transformation of the germ $f:(\&, 0) \rightarrow(\mathbb{C}, 0)$ (see, e.g., [1]). Then one can see that

$$
\zeta_{f}(t)=\widetilde{P}_{\delta, C}\left(T_{1}, \ldots, T_{S}\right)_{T_{i} \mapsto t^{m_{i}}}
$$

where $m_{i}$ is the intersection number of the strict transform of the curve $C$ with the component $E_{i}$ of the exceptional divisor $\mathcal{D}$, in other words $m_{i}$ is the number of components of the strict transform of the curve $C$ which intersects the component $E_{i}$.

\section{References}

[1] Arnold, V. I., Gusein-Zade, S. M., Varchenko, A. N., Singularities of Differentiable Maps, Vol. II. Monogr. in Math. 83, Birkhäuser, Boston-Basel-Stuttgart 1988. Z Zbl 0659.58002 MR 0966191

[2] Campillo, A., Delgado, F., Gusein-Zade, S. M., Integrals with respect to the Euler characteristic over the space of functions and the Alexander polynomial. Proc. Steklov Inst. Math. 238 (2002), 134-147. Zbl 1030.32021 MR 1969310

[3] Campillo, A., Delgado, F., Gusein-Zade, S. M., The Alexander polynomial of a plane curve singularity via the ring of functions on it. Duke Math. J. 117 (1) (2003), 125-156. Zbl 1028.32013 MR 1962784 
[4] Campillo, A., Delgado, F., Gusein-Zade, S. M., The Alexander polynomial of a plane curve singularity and integrals with respect to the Euler characteristic. Internat. J. Math. 14 (1) (2003), 47-52. Zbl 01911965 MR 1955509

[5] Campillo, A., Delgado, F., Gusein-Zade, S. M., Poincaré series of a rational surface singularity. Invent. Math. 155 (2004), 41-53. Zbl 02078249 MR 2025300

[6] Eisenbud, D., Neumann, W., Three-dimensional link theory and invariants of plane curve singularities. Ann. of Math. Stud. 110, Princeton University Press, Princeton, NJ, 1985. Zbl 0628.57002 MR 0817982

[7] Milnor, J., Singular points of complex hypersurfaces. Ann. of Math. Stud. 61, Princeton University Press, Princeton, NJ, 1968. Zbl 0184.48405 MR 0239612

[8] Pinkham, H., Singularités rationelles de surfaces. In Séminaire sur les singularités des surfaces, Lecture Notes in Math. 777, Springer-Verlag, Berlin-Heidelberg-New York 1980, 147-178. Zbl 0459.14009 MR 0579026

Received May 30, 2003

A. Campillo, F. Delgado, University of Valladolid, Department of Algebra, Geometry and Topology, 47005 Valladolid, Spain

E-mail: campillo@agt.uva.es; fdelgado@agt.uva.es

S. M. Gusein-Zade, Moscow State University, Faculty of Mathematics and Mechanics, Moscow, GSP-2, 119992, Russia

E-mail: sabir@mccme.ru 